\title{
Postoperative and Postpartum Onset of Chronic Parkinsonism: Four Case Reports
}

\author{
Manish Ramani B.,' Marcie Rabin L.,' Roger Kurlan.'
}

\begin{abstract}
Background: Certain environmental exposures have been linked to the development of parkinsonism. We report four cases in which the onset of chronic parkinsonism occurred immediately or soon after surgery or childbirth. Results: Exposure to certain anesthetic agents in susceptible individuals or the physiological changes associated with surgery or childbirth may have contributed to or precipitated the development of parkinsonism. Conclusion: Clinicians should be aware that postoperative or postpartum settings are potential precipitants of chronic parkinsonism. More research is needed to clarify contributing factors.
\end{abstract}

Keywords: Parkinsonian disorders, Postoperative period, Postpartum period, Parkinson's disease secondary, Anesthesia (Source: MeSH, NLM).

\section{Introduction}

About the Author: $D r$. Manish Ramani is a graduate of St. George's University School of Medicine, Grenada, West Indies, Boston University school of Medicine in Boston, Massachusetts and Rutgers, The State University of New Jersey in New Brunswick, New Jersey. He is a recipient of the Ruth and WiIliam Silen, M.D. Honorable Mention Award taking 4th Place out of 120 presenters for the best presentation of their poster at The New England Science Symposium at Harvard Medical school.

Submission: Dec. 7, 2013 Acceptance: Jan. 18, 2014 Process: Peer-reviewed

\section{The Cases}

For most patients with Parkinson's disease (PD) and related parkinsonian conditions the underlying etiology is unknown. We now report four cases of parkinsonism observed between 2010 and 2012 during the postoperative or postpartum period that raises important questions about possible precipitating factors, particularly, recently administered anesthesia. None of the four patients had a family history of PD or other parkinsonian like disorders or a known exposure to an identified environmental risk factor for PD. Moreover, in neither case did the patient receive dopamine blocking drugs during anesthesia or for nausea postoperatively.

Case 1. A 43-year-old woman, with no significant past medical history, underwent a surgical replacement of her right hip due to osteoarthritic degeneration. The procedure was performed under spinal anesthesia. The anesthetic agent used is unknown. There were no initial complications, but three days postoperatively she noted the onset of a mild intermittent resting tremor of both legs and constipation. Six months after the onset of symptoms, she was referred to our movement disorder clinic for evaluation and found to have hypomimia, hypophonia, mild resting tremor of both legs, greater on the right, bilateral rigidity, also greater on the right leg, and moderate generalized bradykinesia. She had a slow shuffling gait with decreased right arm swing. Retropulsion was present on pull test. Magnetic resonance imaging (MRI) of the brain was normal. She was diagnosed as having PD and treated with selegiline $5 \mathrm{mg} /$ day and amantadine $100 \mathrm{mg} /$ day which improved her symptoms. Over a period of 2 years her symptoms slowly progressed, but responded well to the addition of carbidopa/ levodopa (30omg/day).

Case 2. A 52-year-old woman, with no past medical history, underwent left brachioplasty surgery under general anesthesia without complication. Agents used during anesthesia included desflurane, propofol, lidocaine, fentanyl, midazolam and rocuronium bromide. Approximately one week after surgery, she developed resting tremor in the left hand. She was evaluated three months later in our clinic and found to have mild resting tremor of the left hand, mild rigidity of the left arm and leg, and slight rigidity of the right arm and leg. Finger taps were slow on the left. She had mild generalized bradykinesia. Her gait was normal except for decreased left arm swing. DaTscan showed that radioligand uptake was nearly absent in the right putamen and mildly reduced in the right caudate and left putamen, suggestive of PD. Her symptoms responded well to treatment with carbidopa/levodopa (300mg/day). Her course has been slowly progressive but responsive to dopaminergic medication over an observation period of 3 years.

Case 3. A 62-year-old man with hepatitis C-associated hepatic failure underwent a liver transplantation under general anesthesia without complications. No information about the anesthetic agents used is available. Upon awakening from surgery, he was found to have dysarthria, micrographia, mild rigidity, and decreased dexterity in his right upper extremity without a tremor, leading to a diagnosis of parkinsonism. Over the next eleven years, his symptoms were well controlled with gradually increasing doses of carbidopa/levodopa/entacapone and pramipexole. At this time he presented to our clinic with painful

${ }^{1}$ Movement Disorders Program, Atlantic Neuroscience Institute, Overlook Medical Center, Summit, NJ 07901 
dystonic dyskinesias involving his feet, neck, shoulders and back, and wearing off motor fluctuations. These symptoms improved following adjustments of medication doses, timing, and physical therapy.

Case 4. A 36-year-old pregnant woman with no past medical history received epidural anesthesia with bupivacaine $0.0625 \%$ at the time of vaginal delivery of her second child. This was poorly tolerated and produced a sensation of whole-body numbness. About 8 weeks after delivery, she developed an action tremor of her right foot when crossing her legs. Over time, she developed tremor of her head, arms and trunk, micrographia and a slow, unsteady and shuffling gait. On physical examination, she had tremor of the head, mild postural and intention tremors of the right hand, mild bilateral rigidity (right greater than left) and dragging of the right leg with decreased bilateral arm swing when walking. Both MRI and fluorodopa PET scanning of the brain were normal. Over the next three years, she experienced progressive parkinsonism that has responded well to carbidopa/levodopa therapy (300mg/day).

\section{Discussion}

In each of the four cases, the patient developed persistent parkinsonism following surgery or childbirth with concomitant exposure to anesthetic agents either during general or spinal anesthesia. Unfortunately in two of the cases, despite our efforts, we were unable to obtain documentation of the specific anesthetic agents administered. Two of the patients had an atypical parkinsonian feature of mainly action tremor or no tremor. In one case, functional imaging demonstrated evidence of striatal dysfunction characteristic of PD and in another functional neuroimaging was normal. The two other patients did not undergo functional neuroimaging.

There has been a prior case report of a patient who presented with acute parkinsonism and dystonia during emergence from general anesthesia.' About 18 months later typical features of PD appeared. Postoperative generalized rigidity can occur as part of anesthetic-induced malignant hyperthermia and it can develop in an isolated fashion after the administration of drugs used in anesthesia, including fentanyl, sufentanil, and droperidol..$^{1-4}$ There are also reports of postoperative rigidity occurring in patients known to have PD, especially after the administration of fentanyl and droperidol.., 3-5 Exposure to the induction agent thiopental and the inhalational anesthetics halothane and isoflurane in animals has been associated with impaired release and transport of dopamine by synaptosomes, 6,7 effects that might induce parkinsonian features in patients. None of these drug effects, however, would be expected to induce long lasting parkinsonism as seen in our cases. ${ }^{8}$ An additional report of the appearance of parkinsonism in a pregnant woman exists. ${ }^{9}$ In this patient, onset was during the eleventh week of gestation and the parkinsonism resolved completely after spontaneous abortion. Women with PD have been reported to experience a worsening of symptoms during pregnancy. ${ }^{10}$

The mechanisms by which surgery, childbirth, or exposure to anesthesia might induce persistent parkinsonism are unclear. It is possible that certain anesthetic agents might induce neurotoxicity in susceptible individuals and this is a concern that deserves further attention. According to one paper, sevoflura- ne, a commonly used anesthetic, causes apoptosis and accumulation of beta-site amyloid precursor protein-cleaving enzyme and beta amyloid in vitro and in vivo, possibly promoting Alzheimer disease neuropathogenesis." A similar mechanism could occur with the development of a parkinsonism condition. Another possible mechanism is the autoantibody-mediated neurotoxicity due to stress, such as the Lupus anticoagulant found in four patients who developed Parkinson's disease post surgery. ${ }^{12}$ Stress secondary to surgery could bring about a condition that promotes neurotoxicity to the dopaminergic neurons. More likely, however, it appears that anesthetic agents or perhaps the stress associated with surgery or childbirth can precipitate clinical expression of an underlying parkinsonian condition that would have otherwise appeared later. It is also possible that subtle, unrecorded physiological changes that can occur during surgery or childbirth, such as anemia, hypotension, anoxia, or immune responses, may have played a role. Finally, the onset of parkinsonism in the setting of surgery or childbirth could have occurred by chance alone, but the close temporal association would be against this possibility.

\section{Conclusion}

In conclusion, each of these four patients developed new-onset parkinsonism without any family history or known environmental exposure. Given the sudden initiation of symptoms in the postoperative or postpartum periods following the administration of an anesthetic, it is prudent for clinicians to be cognizant of these settings as potential precipitants of chronic parkinsonism. Further research is needed to elucidate the factors contributing to this phenomenon, such as a large scale prospective evaluation. 


\section{References}

1. Muravchick S, Smith DS. Parkinsonian symptoms during emergence from general anesthesia. Anesthesiology. 1995;82(1):305-7.

2. Janis KM. Acute rigidity with small intravenous dose of Innovar. A case report. Anesth Analg. 1972;51(3):375-6.

3. Goldberg M, Ishak S, Garcia C, McKenna J. Postoperative rigidity following sufentanil administration. Anesthesiology. 1985;63(2):199-201.

4. Klausner JM, Caspi J, Lelcuk S, Khazam A, Marin C, Hechtman HB, et al. Delayed muscular rigidity and respiratory depression following fentanyl anesthesia. Arch Surg. 1988;123(1):66-7.

5. Easdown LJ, Tessler MJ, Minuk J. Upper airway involvement in Parkinson's disease resulting in postoperative respiratory failure. Can J Anaesth. 1995;42(4):344-7.

6. el-Maghrabi EA, Eckenhoff RG. Inhibition of dopamine transport in rat brain synaptosomes by volatile anesthetics. Anesthesiology. 1993;78(4):750-6. 7. Mantz J, Varlet C, Lecharny JB, Henzel D, Lenot P, Desmonts JM. Effects of volatile anesthetics, thiopental, and ketamine on spontaneous and depola- rization-evoked dopamine release from striatal synaptosomes in the rat. Anesthesiology. 1994;80(2):352-63.

8. Nicholson G, Pereira AC, Hall GM. Parkinson's disease and anaesthesia. Br J Anaesth. 2002;89(6):904-16.

9. Demirkiran M, Aslan K, Bicakci S, Bozdemir H, Ozeren A. Transient parkinsonism: induced by progesterone or pregnancy? Mov Disord. 2004;19(11):1382-4.

10. Kranick SM, Mowry EM, Colcher A, Horn S, Golbe LI. Movement disorders and pregnancy: a review of the literature. Mov Disord. 2010;25(6):665-71.

11. Dong Y, Zhang G, Zhang B, Moir RD, Xia W, Marcantonio ER, et al. The common inhalational anesthetic sevoflurane induces apoptosis and increases beta-amyloid protein levels. Arch Neurol. 2009;66(5):620-31.

12. Chen WH, Lin HS, Kao YF, Lan MY, Liu JS. Postoperative parkinsonism and lupus anticoagulant: a model of autoantibody-mediated neurotoxicity in stress. Brain Inj. 2007;21(5):539-43.

\section{Acknowledgments}

I would like to acknowledge Ayan Sanyal, M.D. a 2nd year resident in Family Medicine at the NEOMED/ Western Reserve Health Education program of Northside Medical Center for his help in completing the final steps of this project.

\section{Conflict of Interest Statement ct Funding}

Dr. Ramani has no outside support and reports no conflicts.

Dr. Kurlan received research support from Otsuka Pharmaceutical Company, LTD, Psyadon Pharmaceuticals, and Rhythm Pharmaceuticals. $\mathrm{He}$ is a consultant for AstraZeneca, PLC. He is also on the speaker's bureau for Teva Pharmaceuticals and UCB Pharma. No conflicts are reported.

Dr. Rabin received research support from Ipsen Pharma. She is on the speaker's bureau for Teva Pharmaceuticals.

Cite as:

Ramani M, Rabin M, Kurlan R. Postoperative and Postpartum Onset of Chronic Parkinsonism: Four Case Reports. Int J Med Students. 2014;2(1):22-4. 